\title{
Iris Template Localization over Internet of Things (IoT)
}

\author{
Ramadan Gad", Ayman EL-Sayed*, Nawal EL-Fishawy ${ }^{*}$, and M. Zorkany ${ }^{* *}$ \\ * Computer Science and Engineering Dept., Faculty of Electronic Engineering, Menoufia \\ University,Egypt.\{ramadangad@el-eng.menofia.edu.eg, ayman.elsayed@el- \\ eng.menofia.edu.eg, nelfishawy@ hotmail.com \} \\ ** Electronic and Communication Engineering Dept., National Telecommunication Institute, \\ Egypt.\{m_zorkany@nti.sci.eg\}
}

(Received: 13 Dec. 2017 - Accepted: 6 Mar. 2018)

\begin{abstract}
Internet of Things (IoT) is growing vastly and survive technology. So; it needs authentication solutions (as iris recognition) to bring safety, and convenience in data and network sharing in the internet of things era. Iris segmentation is most critical stage in the iris recognition system. Some challenges to localize iris such as occlusion by eyelids, eyelashes, and corneal or specular reflection. This paper proposes, a modified algorithm based on masking technique; to localize iris. It solves the limitation of the iris data loss and inconsistencies factors, for capturing conditions and different resolution images. This method gives satisfactory results in factors of accuracy and execution time to be used over IoT. The segmentation success rate is more than $99.545(\%)$, and execution time in worst case $0.758(\mathrm{sec})$. The obtained results improve the efficiency of the proposed iris recognition method and improve IoT security and authentication.
\end{abstract}

Keywords: Internet of Things (IoT); Iris segmentation; Iris localization; Masking technique. 


\section{Introduction}

Internet of Things (IoT) is a concept of connecting any device to the internet. This includes everything has internet access (i.e. monitors, mobile phones, vehicles...etc.). The IoT purpose is to make things smarter and it provides intelligent services [1]. As the IoT continues to grow rapidly from simple systems to complex advanced factories; so security is increasingly important. A fundamental problem that is pervasive in the IoT today - that must be solved - is dealing with security attacks[2].Security is critical to IoT network and the first line of defense against data corruption. Ensuring security (authentication) is critical as the system is automatically linked to actuators and protecting the systems from intruders becomes very important[3].

The utilization of iris based authentication solutions across all of these markets and sectors will add a high-level of security, while keeping systems smart, fast, and easy to use. Iris authentication is the process to confirm a user's identity and is a key factor in securing any IoT device. Iris considered more accurate, reliable, and stable. Some desirable properties like individuality, uniqueness, stability over the human life, and well protection distinguish the iris pattern for human authentication and identification [4, 5]. Based on[6], it is the lowest recognition error rate; so it is the best choice in different potential applications that need high security. Iris overview and iris system components, benefits, drawbacks, and challenges are in details discussed in[7].

In this paper, Iris Identification mechanism for authentication and authorization - as well as provenance of information, ownership and "market mechanisms" for information - become particularly important in IoT environment. The iris recognition system is divided into four main stages, after iris capturing: iris segmentation, normalization, feature extraction and template matching [5][8].An iris segmentation is the most critical step, which includes inner and outer location. Unfortunately, some challenges face iris segmentation as corneal/specular reflections, upper and lower eyelids, and eyelashes occlusion[9]. It is reported that inaccurate iris segmentation leads to failure in matching result. Generally, iris segmentation is time consuming and sensitive to noise[4]. In addition, most of traditional techniques like Integrodifferential operator[10] and Circular Hough Transform (CHT) [7] have 
complex computations and large memory occupation[5]. This have a bad effect on bandwidth and server response over iris based IoT authentication system.

All of the above challenges make the segmentation so difficult and urgent. Therefore, one of the main objectives in this research, is to implement an accurate automated segmentation algorithm suitable for IoT servers and architecture. It merges iris segmentation and normalization in one point. Likewise, get rid of the noise and undesired artifacts mentioned without affecting the iris region structure. The proposed algorithm will be tested by four famous and recent benchmark datasets for all most capturing conditions and different resolution. They are CASIA v.1, CASIA v.4-Interval, SDUMLA-HMT, and UBIRIS v.1.

This paper is organized as follows, related methods as previous work is introduced in section II. The suggested approach to localize and normalize the iris are illustrated in details in section III. Accordingly, section IV presents the results and discussion. The benefits of our methodology will be discussed in the same division. Lastly, section V, concludes our research work.

\section{Related Work}

The IoT is extremely vulnerable to attacks. Many solutions (like cryptography and key management) have been proposed in the recent past. But few researches in iris recognition methods for IoT security. Regarding to segmentation step, many specular reflection removal, and pupil/iris detection methods proposed in the area. Some limitations are in these methods. Zhaofeng He et.al.[4], gave good results with the MMU iris database but, it didn't with CASIA iris database V3. Ann A. Jarjes et.al.[11], iris structure was totally modified so; it is unsuitable to generate the iris template. In addition, in order to fill all dark points, this technique sweeps all image without focus on the region of interest. As a result, in my opinion, a waste of time for nothing. Moreover, Nguyen Thanh et.al.[12], consolidated shrinking and expanding active contour methods when localizing inner and outer iris boundaries. 
A mathematical algorithm by Walid Aydi et.al.[13], to fill the pupil speckles, provides faster and less harm to iris structure. But, they did not consider its validity to the pupil location. Chunxian Song et.al.[9], Pengfei Cai et.al. [14], and Yuhui Lin et.al. [8], algorithms failed to segment some pupil region cases due to the speckles in pupil area near the boundary, and the heavy upper or lower eyelid covered the iris. Circular masking technique implemented by Mahesh Patil et.al.[15]. It was lack to normalization processes. In Safaa S Omran et.al.[16], CHT and rubber sheet model used to localize CASIA v.1 dataset images. It needs more time on CPU execution. Saiyed Umer et.al.[17], produced constrained CHT to localize uni-modal iris images taken from four datasets (CASIA v.3, MMU1, UPOL, and IITD). It suffers from complexity and more iterations.

In[6], [7], mixed algorithms were described to implement an iris recognition system based on CASIA v.1 only. CHT with modifications applied to isolate the iris. Hough transform is unaffected by noise and it provides good accuracy in localization. But, it is computationally exhaustive. It failed to detect some circles; since it depends on a threshold values. In order to solve the performance limitations of these previous methods; a new method in [18] proposed. But, this algorithm faces a limitation of mask size. Moreover, a large amount of iris template pixels lost, especially when the pupil size is small. This will decrease the scalability. Also, it does not consider the rotation or camera distance. This paper proposes a modified masking technique; to overcome these issues.

\section{The Proposed Algorithm}

Generally, iris recognition method should be introduced by scanning and encrypting users' 'iris signature'; to provide a form of biometric authentication over IoT. The approach provides iris recognition based biometric system in where the camera takes images of persons and sends it to the server over internet for further processing and storing, for future usages. Camera like infrared camera, webcam, camera of mobile phone, USB Camera could be used. IRIS server is used for data storing, processing and analyzing for iris recognition process. The iris identification server must have iris codes of all users in data base. The basic diagram of overall iris recognition system over IoT is illustrated in Fig. 1. 
In this paper, the iris templates (iris codes) are stored in data base server that will be used later for comparing with the next queries of person for authentication. If the enrolled iris gets matched with the stored one, then the message goes to the administrative to use IoT applications. If not matched, then prevent this user. Segmentation step, as the more critical in the recognition operation, is proposed. Fig. 2, describes the steps of the proposed method. In the following subsections we will illustrate the suggested method to generate iris template stored in IoT server in details.

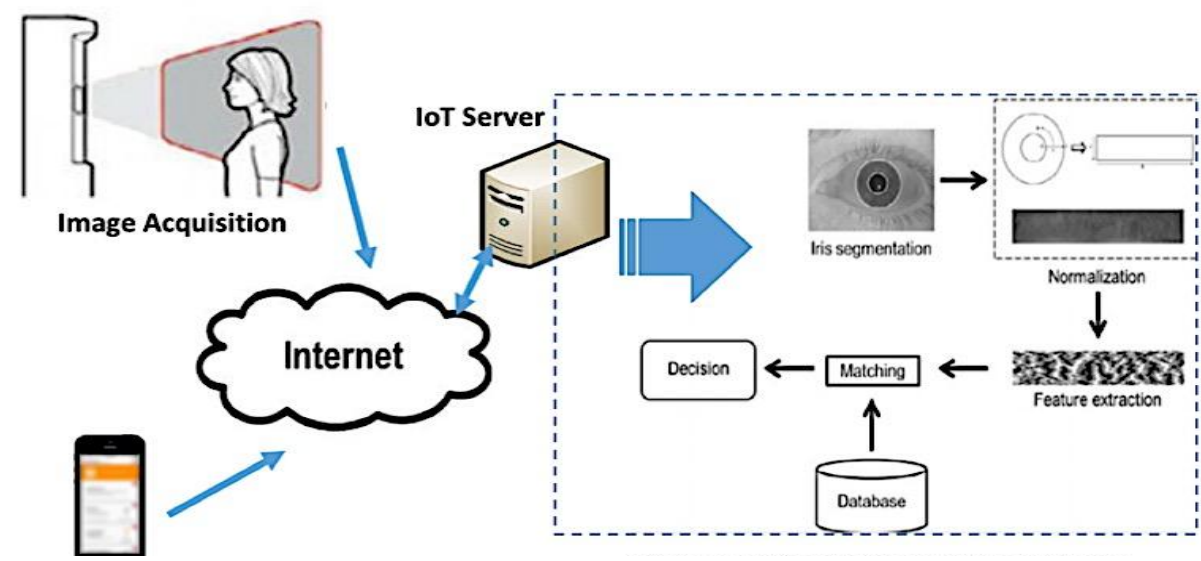

Fig.1. A block diagram of an iris recognition system over IoT.

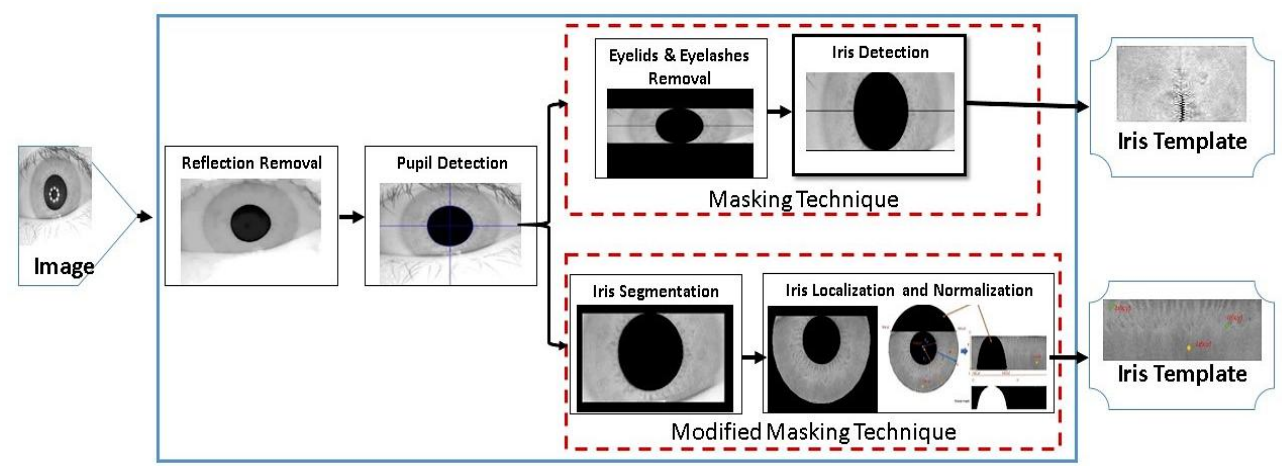

Fig.2. The block diagram of the proposed iris localization algorithm. 


\subsection{Reflection Removal and Pupil Detection}

In [18], a sequence of morphological operations proposed; to remove the corneal and specular reflections in iris images. Regarding pupil detection, Adaptive Local Threshold (ALT)algorithm depending on mean filter is used to filter bright pixels in the iris image $\mathrm{A}(\mathrm{x}, \mathrm{y})$. Regards the result binary image, shown in Fig. 3, let $R_{\text {matrix }}$ is the summation matrix for each row, and $C_{\text {matrix }}$ is the summation matrix for each column. The row-centroid $\left(\mathrm{R}_{\mathrm{c}}\right)=$ Index $\left(\max \left|R_{\text {matrix }}\right|\right)$, and column-centroid $\left(\mathrm{C}_{\mathrm{c}}\right)=\operatorname{Index}\left(\max \left|C_{\text {matrix }}\right|\right)$. The 'Index' parameter is the position (coordinate) of the pixel in image along $\mathrm{x}$-axis and $\mathrm{y}$-axis. The sign '|.|' means the absolute value. The pupil center point $\left(\mathrm{P}_{\mathrm{c}}(\mathrm{x}, \mathrm{y})\right)$ is the intersection point of $\mathrm{R}_{\mathrm{c}}$ and Cc. The pupil radius $\left(R_{p}\right)$ calculated as:

$$
\begin{aligned}
& R_{p}=\max \left(R_{p 1}, R_{p 2}, R_{p 3}, R_{p 4}\right) \\
& R p_{1}=\left|\operatorname{Index}\left(P_{1}(x, y)\right)-\operatorname{Index}\left(P_{c}(x, y)\right)\right| \\
& R p_{2}=\left|\operatorname{Index}\left(P_{2}(X, y)\right)-\operatorname{Index}\left(P_{c}(X, y)\right)\right| \\
& R p_{3}=\left|\operatorname{Index}\left(P_{3}(X, y)\right)-\operatorname{Index}\left(P_{c}(x, y)\right)\right| \\
& R p_{4}=\left|\operatorname{Index}\left(P_{4}(X, Y)\right)-\operatorname{Index}\left(P_{c}(X, Y)\right)\right|
\end{aligned}
$$

Each of the points $\mathrm{P}_{1}, \mathrm{P}_{2}, \mathrm{P}_{3}$, and $\mathrm{P}_{4}$ has the index of the first zero-value pixel along the radius axis in the four directions. Pupil detection steps with the results are illustrated in Fig. 4 , in sequence. The pupil region mask $\left(\mathrm{M}_{\mathrm{p}}(\mathrm{x}, \mathrm{y})\right)$ (Fig. 4-d) identified by the center $\left(\mathrm{P}_{\mathrm{c}}(\mathrm{x}, \mathrm{y})\right)$ and the radius $\left(R_{p}\right)$ is multiplied again in the original image $\mathrm{A}(\mathrm{x}, \mathrm{y})$; to isolate the iris region $\mathrm{I}(\mathrm{x}, \mathrm{y})$ free of artifacts without deformation. Pupil border is shown in Fig. 4-f.

\subsection{Masking Technique (MT)}

Let iris image $\mathrm{I}(\mathrm{x}, \mathrm{y})$ has $\mathrm{m} \times \mathrm{n}$ pixels, $\forall 1 \leq y \leq n$, the eyelashes/eyelids removing mask $\left(\mathrm{M}_{\mathrm{e}}(\mathrm{x}, \mathrm{y})\right)$ identified as:

$$
M_{e}(x, y)=\left\{\begin{array}{c}
0: 1 \leq x \leq \text { Index }\left(P_{4}(x, y)\right), \text { Index }\left(P_{2}(x, y)\right) \leq x \leq m \\
1: \text { Index }\left(P_{4}(x, y)\right)<x<\operatorname{Index}\left(P_{2}(x, y)\right),
\end{array}\right.
$$

The final mask $\left(\mathrm{M}_{\mathrm{O}}(\mathrm{x}, \mathrm{y})\right)$, is shown in Fig. 5-a, identified in binary format as:

$$
M_{o}(x, y)=M_{p}(x, y) \times M_{e}(x, y)
$$


In [18], a fixed template size (60x90 pixel) generated. This was unsuitable for some images in datasets; due to images size and resolution. Some modification done over MT; the N pixels to the left and right of the localized pupil are concatenated. Iris template is created by mapping the selected pixels on a fixed size $(60 \times 2 \mathrm{~N})$ matrix. see Fig. $5(\mathrm{~b}-\mathrm{c})$.

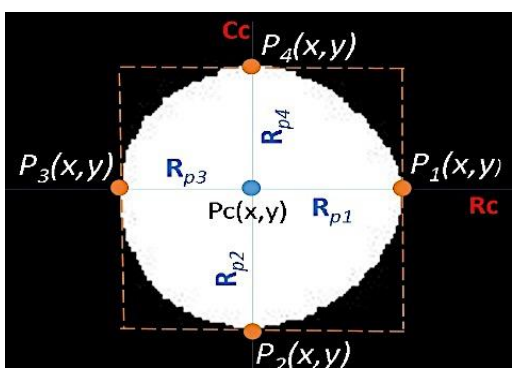

Fig.3. Pupil parameters detection (pupil center $\mathrm{P}_{\mathrm{c}}(\mathrm{x}, \mathrm{y})$ and pupil radius $\mathrm{R}_{\mathrm{p}}$ ).

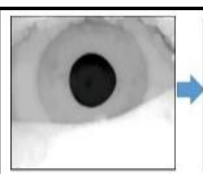

(a)

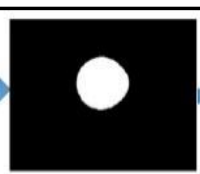

(b)

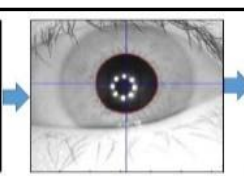

(c)

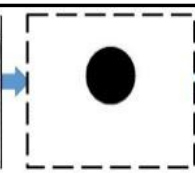

(d)

(a) Reflection removal result image.

(b) Pupil after adaptive threshold.

(c) Pupil circle parameters (Pc, Rp).

(d) The mask of pupil region.

(e) Original image.

(f) Punil segmentation free of reflections.

Fig.4. Pupil detection steps.

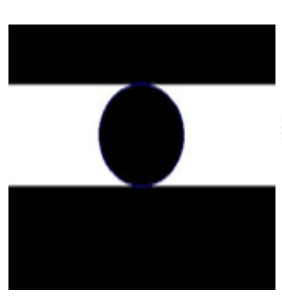

(a)

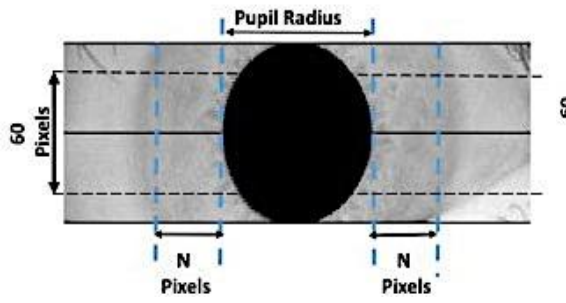

(b)

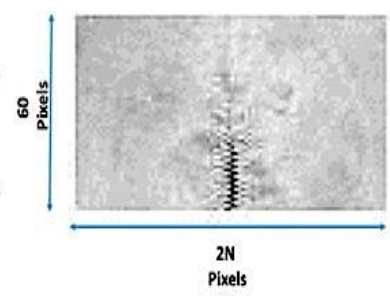

(c)

Fig.5. MT processes illustration. (a) Iris mask $\mathrm{M}_{0}(\mathrm{x}, \mathrm{y})$ by aid of pupil parameters and eyelashes mask. (b) Iris template parameters declaration. (c) Final iris template. 


\subsection{Modified Masking Technique (MMT)}

This algorithm is a modification for MT[18]. Let us call it Modified Masking Technique (MMT) for future citation. It consists of sequential steps as follows:

Step 1-Mask generation: Let iris image $\mathrm{I}(\mathrm{x}, \mathrm{y})$ with size $\mathrm{m} \times \mathrm{n}$, the eyelashes removing mask $\left(\mathrm{M}_{\mathrm{e}}(\mathrm{x}, \mathrm{y})\right)$ updated as[18]:

$$
M_{e}(x, y)=\left\{\begin{array}{c}
0: 1 \leq x \leq \operatorname{Index}\left(P_{4}(x, y)\right), \forall 1 \leq y \leq n \\
1: \operatorname{Index}\left(P_{4}(x, y)\right)<x \leq m, \forall 1 \leq y \leq n
\end{array}\right.
$$

The mask updated sequentially, as shown in Fig. 6, related to each process as in the next steps. The occlusion mask $\left(\mathrm{M}_{\mathrm{O}}(\mathrm{x}, \mathrm{y})\right)$ identified in binary format as in equation (7).

Step 2- Iris parts detection: Let $M$ be a unit vector with (n) length. From point $\mathrm{p}_{1}(\mathrm{x}, \mathrm{y})$ along the pupil axe till the end of image $\mathrm{I}(\mathrm{x}, \mathrm{y})$ with length $(\mathrm{L})$. Accumulated vector $\left(\mathrm{V}_{\text {right }}\right)$ generated as a result of convolving the image $\mathrm{I}(\mathrm{x}, \mathrm{y})$ by this binary mask $(\mathrm{M})$. The right border point separates iris-sclera is $\left(\mathrm{I}_{\mathrm{R}}\right)$ defined as $\mathrm{I}_{\mathrm{R}}=\operatorname{Index}\left(\max \left(\mathrm{V}_{\text {right }}\right)\right)$. As well as the left border $\left(\mathrm{I}_{\mathrm{L}}\right)$ defined by the same manner $\mathrm{I}_{\mathrm{L}}=\operatorname{Index}\left(\max \left(\mathrm{V}_{\text {left }}\right)\right)$ in the opposite side. Both $\mathrm{V}_{\text {right }}$ and $\mathrm{V}_{\text {left }}$ are calculated as:

$$
\begin{array}{r}
V_{\text {right }_{\mid j}}=\sum_{i=1}^{n} M_{i} * I\left(x_{j}, y_{k}\right): \operatorname{index}\left(P_{1}(x, y)\right)<j<L, \forall j+i-1< \\
k<j+n-1 \\
V_{\text {left }_{\mid j}}=\sum_{i=1}^{n} M_{i} * I\left(x_{j}, y_{k}\right): 0<j<\operatorname{index}\left(P_{3}(x, y)\right), \forall j+i-1<k< \\
j+n-1
\end{array}
$$

Mask convolution operations are illustrated in Fig. 7; to detect the right iris border. The result processed image $\mathrm{I}(\mathrm{x}, \mathrm{y})$ with size $\mathrm{m} \times \mathrm{n}$, produced as multiplication of original image $A(x, y)$ by the new mask $\left(M_{m}(x, y)\right)$ identified as:

$$
\begin{aligned}
& M_{m}(x, y)=M_{O}(x, y) \times M_{L R}(x, y) \\
& M_{L R}(x, y)=\left\{\begin{array}{c}
0: 1 \leq y \leq I_{L}, I_{R} \leq y \leq n, \forall 1 \leq x \leq m \\
1: I_{L}<y<I_{R}, \forall 1 \leq x \leq m
\end{array}\right.
\end{aligned}
$$

Step 3- Eyelid detection: Firstly, let $\mathrm{I}_{\mathrm{bin}}(\mathrm{x}, \mathrm{y})$ be the binary image result as applying the local average-based ALT filter on A(x,y). Secondly, apply the convolution by mask $(M)$. From point $\mathrm{p}_{2}(\mathrm{x}, \mathrm{y})$ along the pupil axe till the end of $\mathrm{N}$ length image $\mathrm{I}_{\mathrm{bin}}(\mathrm{x}, \mathrm{y})$, The bottom border point separates iris-eyelid is 
$\left(\mathrm{I}_{\mathrm{B}}\right)$ defined as $\mathrm{I}_{\mathrm{B}}=\operatorname{Index}\left(\max \left(\mathrm{V}_{\mathrm{B}}\right)\right)$ identifies the mask $\mathrm{M}_{\mathrm{B}}(\mathrm{x}, \mathrm{y}) . \mathrm{V}_{\mathrm{B}}$ is calculated as:

$V_{B_{\mid j}}=\sum_{i=1}^{n} M_{i} * I_{b i n}\left(x_{j}, y_{k}\right): \operatorname{Index}\left(P_{2}(x, y)\right)<j \leq N, \forall j+i-1<k<$

$$
j+n-1
$$

The result processed image $\mathrm{I}(\mathrm{x}, \mathrm{y})$ with size $\mathrm{m} \times \mathrm{n}$, produced as multiplication of original image $\mathrm{A}(\mathrm{x}, \mathrm{y})$ by the new mask $\left(\mathrm{M}_{\mathrm{E}}(\mathrm{x}, \mathrm{y})\right)$ identified as:

$$
\begin{aligned}
& M_{E}(x, y)=M_{m}(x, y) \times M_{B}(x, y) \\
& M_{B}(x, y)=\left\{\begin{array}{l}
0: I_{B} \leq x \leq m, \forall 1 \leq y \leq n \\
1: 1 \leq x<I_{B}, \forall 1 \leq y \leq n
\end{array}\right.
\end{aligned}
$$

Step 4- Extract iris: At this point, three points derived in iris mask $\left(\mathrm{I}_{\mathrm{R}}, \mathrm{I}_{\mathrm{L}}\right.$, and $\left.\mathrm{I}_{\mathrm{B}}\right)$ as shown in Fig. 8-a, with three distances $\left(\mathrm{R}_{\text {dist }}, \mathrm{L}_{\text {dist }}\right.$, and $\left.\mathrm{Y}_{\text {dist }}\right)$ where:

$$
\begin{aligned}
& R_{\text {dist }}=R_{p 1}+\left(\left|\operatorname{Index}\left(I_{R}(x, y)\right)-\operatorname{Index}\left(P_{1}(x, y)\right)\right|\right) \\
& L_{\text {dist }}=R_{p 3}+\left(\left|\operatorname{Index}\left(I_{L}(x, y)\right)-\operatorname{Index}\left(P_{3}(x, y)\right)\right|\right) \\
& Y_{\text {dist }}=R_{p 2}+\left(\left|\operatorname{Index}\left(I_{B}(x, y)\right)-\operatorname{Index}\left(P_{2}(x, y)\right)\right|\right)
\end{aligned}
$$

In some cases, $\mathrm{R}_{\mathrm{dist}} \neq \mathrm{L}_{\text {dist }}$; due to boundary detection factors, and of course the third parameter $\left(\mathrm{Y}_{\text {dist }}\right)$ is not equal to any of them; due to occlusion. In addition, some unwanted parts of sclera still find as noise. The mask in this case is an ellipse $\mathrm{M}_{\mathrm{S}}(\mathrm{x}, \mathrm{y})$ (see Fig.8-b) identified by the pupil center $\mathrm{P}_{\mathrm{c}}(\mathrm{x}, \mathrm{y})$ and two radii. One of them is $\mathrm{Y}_{\text {dist. }}$ The other radius $\mathrm{X}_{\text {dist }}$ defined as:

$$
X_{\text {dist }}=\min \left(R_{\text {dist }}, L_{\text {dist }}\right)
$$

The final mask $\left(\mathrm{M}_{\mathrm{I}}(\mathrm{x}, \mathrm{y})\right)$ used to localize the iris boundary generated in binary format identified as:

$$
M_{I}(x, y)=M_{S}(x, y) \times M_{E}(x, y)
$$

The result of mask processing to localize the iris is shown in Fig. 8(c-f).

Step 5- Normalize iris: As we concentrate on the pupillary zone, the shape of generated mask could be approximated to a circle by using an iris radius $\mathrm{R}_{\mathrm{i}}=$ min $\left(\mathrm{X}_{\text {dist }}, \mathrm{Y}_{\text {dist }}\right)$ as iris radius. By using the pupil center $\mathrm{P}_{\mathrm{c}}(\mathrm{x}, \mathrm{y})$ as reference point, pupil radius $R_{p}$, and iris radius $R_{i}$, rubber sheet model[10] used to treat the inconsistencies invariant. The image $\mathrm{I}(\mathrm{x}, \mathrm{y})$ transformed from the Cartesian coordinate $(\mathrm{x}, \mathrm{y})$ to the polar one $(\mathrm{r}, \theta)$. As shown from Fig. 9, full part of iris generated by using $\theta=(0-2 \pi)$. 


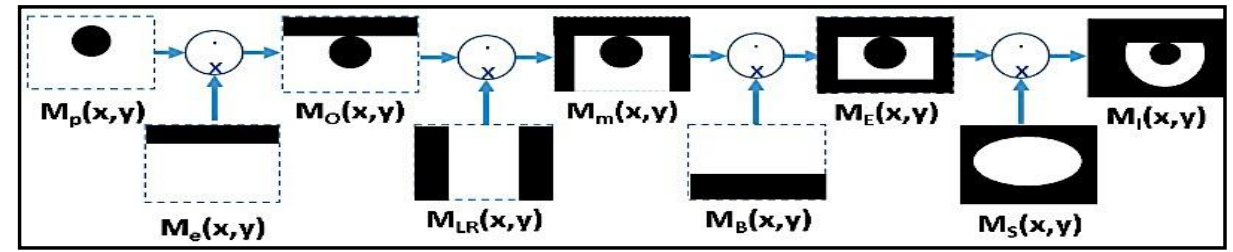

Fig.6.The mask generation steps to localize the iris in MMT.

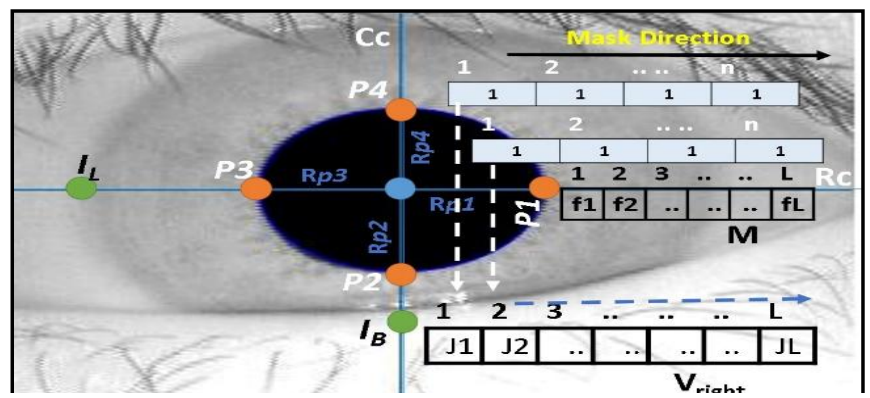

Fig.7. The idea of iris borders detection by using mask operations.

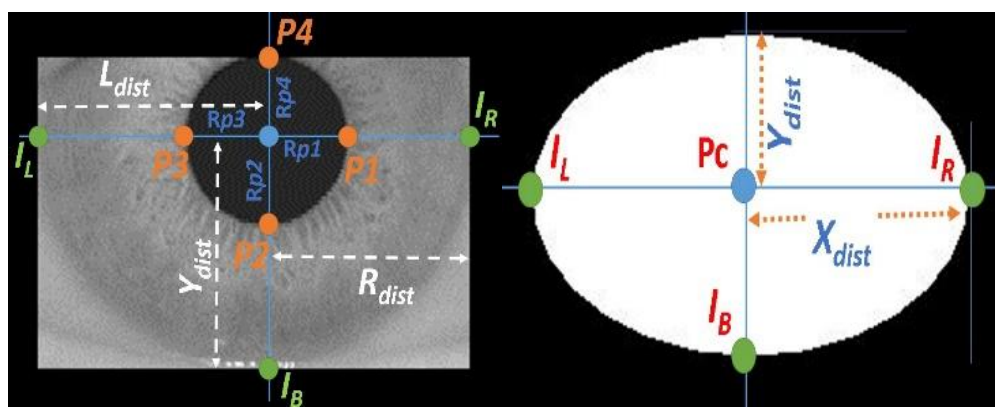

(a)

(b)

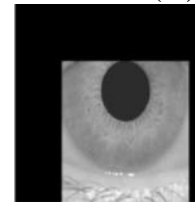

(c)

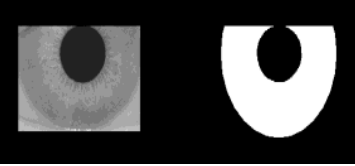

(d)

(e)

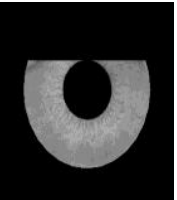

(f)

Fig.8. Iris mask derivation process with results. (a) Iris radius calculation. (b) $\mathrm{M}_{\mathrm{S}}(\mathrm{x}, \mathrm{y})$ calculation. (c-f) The right and left iris borders detection. 


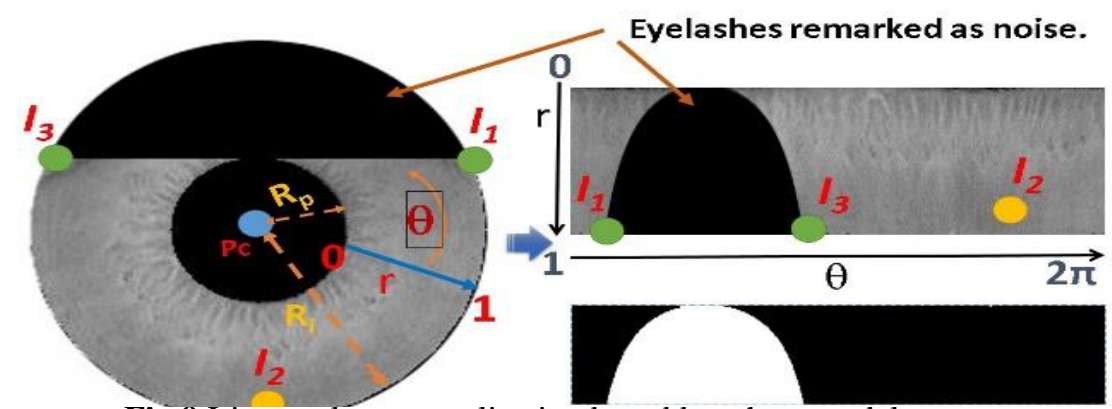

Fig.9.Iris template normalization by rubber sheet model.

\section{Results and Discussion}

The performance of the previously discussed method tested, using MATLAB (2009a), installed on PC processor Dual-Core 1.6 GHZ/2MB Cache, RAM 2GB. In these results, four well-known iris databases acquired in different criteria are used. They are briefly summarized in Table 1 .

-Evaluation metrics: The metrics used to evaluate the pupil and iris segmentation in this paper as:

(a) Structural Similarity Index (SSIM): The overall quality assessment index is a multiplicative combination of the luminance, the contrast, and the structural term computations. SSIM for two images is identified as[19]:

$$
\operatorname{SSIM}(x, y)=\frac{\left(2 \mu_{x} \mu_{y}+C_{1}\right)\left(2 \sigma_{x y}+C_{2}\right)}{\left(\mu_{x}^{2}+\mu_{y}^{2}+C_{1}\right)\left(\sigma_{x}^{2}+\sigma_{y}^{2}+C_{2}\right)}
$$

Where $\mu_{\mathrm{x}}, \mu_{\mathrm{y}}, \sigma_{\mathrm{x}}, \sigma_{\mathrm{y}}$, and $\sigma_{\mathrm{xy}}$ are the local means, standard deviations, and cross-covariance for images $\mathrm{x}$, and $\mathrm{y}$. Constants $\mathrm{C} 1=\mathrm{C} 2=0$ for the universal quality index.

(b) Mean Square Error (MSE): Computes the average of squared intensity differences of corrupted and original image pixels as[20]:

$$
M S E=\frac{1}{m n} \sum_{i=0}^{m-1} \sum_{j=0}^{n-1}(X(i, j)-Y(i, j))^{2}
$$

Where $X(i, j)$ and $Y(I, j)$ are the two images with size $m \times n$ pixels. 
(c) Image Quality Index: It depends on luminance distortion, contrast distortion, and loss of correlation factors. The $\mathrm{Q}$ index is the product of them all as[21]:

$$
Q=\left(\frac{\sigma_{x y}}{\sigma_{x} \sigma_{y}}\right)\left(\frac{2 \bar{x} \bar{y}}{(\bar{x})^{2}+(\bar{y})^{2}}\right)\left(\frac{2 \sigma_{x} \sigma_{y}}{\sigma_{x}^{2}+\sigma_{y}^{2}}\right)
$$

Its range of values between $[0,1]$, where the value 1 means the best quality for all.

(d) Accuracy (success rate): the percentage number of successful segmented images per total original enrolled. any loss of iris part in the segmentation process, in turn, weakens the very useful unique iris features, which are essential for differentiating inter- class subjects, thus adversely affects the recognition performance of overall system [22].

(e) Execution time: total time required to obtain segmented iris image from resized grayscale image.

These evaluation metrics used in test defined in details with in [18].

-Simulation results: With the proposed algorithm, the accuracy rate of pupil location reaches $99.69 \%$ without speckles in all datasets images. It is better accuracy rate than some other previous methods as declared in Table 2.Regarding MT, the variable parameter (N) controls the template size $(60 \times 2 N)$ varied in range [30-60] pixels. The success rate recorded according each benchmark for each value of $\mathrm{N}$ in Table 3. In MMT, the mask size (n) controls the iris region. In Table 4, the accuracy changed according to the value of $(n)$ parameter. SDUMLA-HMT recorded the lowest rate in this metric; as its images are very large $(768 \times 576$ pixels $)$ compared to others.

In addition, the specular reflect in pupil is one circular white spot; our algorithm hardly detects pupil with such environmental nature. It is noticed that, the best result in MT method is when $\mathrm{N}=30$ pixels. When $\mathrm{N}$ is larger, parts from sclera included in iris template as noise. $\mathrm{N}$ may be increased to 45 pixels to collect highest useful information in recognition processes. But, in UBIRIS only, the accuracy will decrease accordingly. Range of (30-35) for mask size (n) in MMT achieves the best accuracy. When $(n<30)$ the final iris mask expanded including sclera pixels gradually. For $(n>35)$, the mask loses 
more information from iris circle. In general, the proposed method is more accurate than CHT [23] as listed in Table 5.

The execution time of each proposed method is listed for each test benchmark used in table 6. MT is faster than MMT; as it needs little operations. The two methods are faster than the CHT due to the iterations the later do. The MMT method takes more computations to localize iris region due to the different noise challenges facing it.

Table 1. Brief description of used databases.

\begin{tabular}{|c|c|c|c|c|}
\hline $\begin{array}{c}\text { Database/ } \\
\text { Specification }\end{array}$ & CASIA v1[24] & $\begin{array}{l}\text { CASIA v4- } \\
\text { interval[25] }\end{array}$ & $\begin{array}{c}\text { UBIRIS } \\
\text { v1[26] }\end{array}$ & $\begin{array}{l}\text { SDUMLA- } \\
\text { HMT[27] }\end{array}$ \\
\hline Description & $\begin{array}{l}756 \text { iris images } \\
\text { from } 108 \text { eyes, } 7 \\
\text { images in two } \\
\text { sessions, } 3 \text { in the } \\
\text { first and } 4 \\
\text { samples in the } \\
\text { second session. }\end{array}$ & $\begin{array}{lr}2,639 & \text { images } \\
\text { from } & 249 \\
\text { subjects, } & 395 \\
\text { classes. images } \\
\text { were captured in } \\
\text { two sessions. }\end{array}$ & $\begin{array}{l}1,877 \\
\text { images from } \\
241 \text { persons } \\
\text { in two } \\
\text { distinct } \\
\text { sessions. }\end{array}$ & $\begin{array}{l}1,060 \text { iris images } \\
\text { from } 106 \\
\text { individuals. Each } \\
\text { subject provided } \\
5 \text { images for each } \\
\text { eye. }\end{array}$ \\
\hline $\begin{array}{l}\text { Image resolution } \\
\text { (pixel) }\end{array}$ & $320 \times 280$ & $320 \times 280$ & $200 \times 150$ & $768 \times 576$ \\
\hline Subjects/images & All & All & All & All \\
\hline
\end{tabular}

Table 2.Accuracy comparison with some other methods of pupil detection.

\begin{tabular}{|c|c|c|c|c|c|}
\hline & $\begin{array}{c}\text { Nguyen Thanh } \\
\text { et.al.[12] }\end{array}$ & $\begin{array}{c}\text { Chunxian } \\
\text { Song } \\
\text { et.al.[9] }\end{array}$ & $\begin{array}{c}\text { Pengfei } \\
\text { Cai } \\
\text { et.al.[14] }\end{array}$ & $\begin{array}{c}\text { Yuhui } \\
\text { Lin } \\
\text { et.al.[8] }\end{array}$ & Proposed \\
\hline $\begin{array}{c}\text { Accuracy } \\
(\%)\end{array}$ & 99.20 & 99.55 & 93.50 & 90.00 & $\mathbf{9 9 . 6 9}$ \\
\hline
\end{tabular}


Table 3. Segmentation success rate for each $\mathrm{N}$ pixels in MT.

\begin{tabular}{|l|c|c|c|c|}
\hline \multirow{2}{*}{$\begin{array}{c}\mathrm{N} \\
\text { pixel })\end{array}$} & \multicolumn{4}{|c|}{ Success rate (\%) for each database } \\
\cline { 2 - 5 } & CASIA v. 1 & CASIA v. 4 & SDUMLA-HMT & UBIRIS v. 1 \\
\hline 60 & 96.179 & 95.724 & 91.935 & 78.268 \\
\hline 55 & 97.453 & 96.998 & 93.209 & 79.542 \\
\hline 50 & 98.726 & 98.271 & 94.482 & 80.815 \\
\hline 45 & 100 & 99.545 & 95.756 & 82.089 \\
\hline 40 & 100 & 99.545 & 95.756 & 87.882 \\
\hline 35 & 100 & 99.545 & 95.756 & 93.674 \\
\hline 30 & $\mathbf{1 0 0}$ & $\mathbf{9 9 . 5 4 5}$ & $\mathbf{9 5 . 7 5 6}$ & $\mathbf{9 9 . 4 6 7}$ \\
\hline
\end{tabular}

Table 4. Segmentation success rate for all datasets according to mask size (n) in MMT.

\begin{tabular}{|l|c|c|c|c|}
\hline \multirow{2}{*}{$\begin{array}{c}\text { Mask Size(n) } \\
\text { (pixel) }\end{array}$} & \multicolumn{4}{|c|}{ Success rate (\%) for each database } \\
\cline { 2 - 5 } & CASIA v. 1 & CASIA v. 4 & SDUMLA-HMT & UBIRIS v. 1 \\
\hline 5 & 95.362 & 90.775 & 82.033 & 74.394 \\
\hline 10 & 96.202 & 91.843 & 83.379 & 75.822 \\
\hline 15 & 97.043 & 92.911 & 84.724 & 77.250 \\
\hline 20 & 97.883 & 93.979 & 86.070 & 78.678 \\
\hline 25 & 98.589 & 96.762 & 90.913 & 85.608 \\
\hline $\mathbf{3 0}$ & 99.294 & $\mathbf{9 9 . 5 4 5}$ & $\mathbf{9 5 . 7 5 6}$ & 92.537 \\
\hline $\mathbf{3 5}$ & $\mathbf{1 0 0}$ & 97.751 & 92.971 & $\mathbf{9 9 . 4 6 7}$ \\
\hline 40 & 98.791 & 95.957 & 90.187 & 93.149 \\
\hline 45 & 97.581 & 94.163 & 87.402 & 86.831 \\
\hline 50 & 96.372 & 92.369 & 84.618 & 80.512 \\
\hline 55 & 95.162 & 90.575 & 81.833 & 74.194 \\
\hline 60 & 94.322 & 89.507 & 80.487 & 72.766 \\
\hline
\end{tabular}

Table 5.Segmentation success rate comparison between CHT and proposed method.

\begin{tabular}{|l|c|c|}
\hline \multirow{2}{*}{ Dataset/Method } & \multicolumn{2}{|c|}{ Success rate(\%) } \\
\cline { 2 - 3 } & CHT + Rubber Sheet Model[7], [23] & Modified Masking Technique \\
\hline CASIA v. 1 & 99.867 & 100 \\
\hline CASIA v. 4 & 82.274 & 99.545 \\
\hline SDUMLA-HMT & 85.645 & 95.756 \\
\hline UBIRIS v. 1 & 82.690 & 99.467 \\
\hline
\end{tabular}


Table 6.Execution time comparison of the proposed algorithms.

\begin{tabular}{|l|c|c|c|}
\hline \multirow{2}{*}{ Dataset/Method } & \multicolumn{3}{|c|}{ Execution time(Sec) } \\
\cline { 2 - 4 } & CHT + Rubber Sheet Model[7], [23] & MT & MMT \\
\hline CASIA v. 1 & 21.630 & 0.0002 & 0.758 \\
\hline CASIA v. 4 & 16.359 & 0.0009 & 0.996 \\
\hline SDUMLA-HMT & 26.480 & 0.0004 & 0.850 \\
\hline UBIRIS v. 1 & 21.630 & 0.0004 & 0.957 \\
\hline
\end{tabular}

-Discussion: The advantages of the proposed approach are: (1) It is fast; this makes it suitable for implementation by many solutions. It is useful in realtime application, (2) It is a simple method, that it removes iris occlusions and reflections with less computations and memory occupation, (3) It Merges iris segmentation and normalization into one stage.In MT, a large amount of iris template pixels lost, especially when the pupil size is small. This will decrease the scalability. Also, it does not consider the rotation or camera distance. These issues solved by MMT. In general, the proposed approach is faster than CHT. In addition, iris localized free of reflections and occlusions without reformation of structure and with no iterations. On other hand, some samples failed to detect the iris region successfully. The causes for iris boundary failure as: (i) iris area is smaller than the size $(60 \times 2 \mathrm{~N}$ pixel) identified in proposed (MT). (ii) If pupil detected wrong; the iris also failed to detect. This could be enhanced by adjusting the mask size (n) parameter and used thresholds.

It is noticed from Table 2, the enhancement in the accuracy factorbetweenNguyen Thanh et.al. [12], Chunxian Song et.al. [9], and the proposed method in this paper is nearly small. But, the from complexity analysis view point this proposed distinguishes by other important factor in real-time application. Based on Table 6, the execution time in worst case of masking technique is $0.0009 \mathrm{sec}$ compared with Nguyen Thanh et.al. [12], Chunxian Song et.al. [9] ( the best case of the execution time in the same database not less than $2.397 \mathrm{sec}$ ). The reason that the proposed masking technique does less iterations and so complexity.

\section{Conclusion}

The paper has presented an automated segmentation technique over internet of things in which an iris recognition system is used. The proposed method 
just need any camera interface and IoT server. As presented, a failure in iris boundary localization, is a vital error pattern matching and identification over IoT server. Consequently, this will affect badly the recognition rate. The proposed isolates the iris free of artifacts without changing its structure. In this proposed method. modified masking technique (MMT) solved the limitation of the iris data loss in Masking Technique (MT) and inconsistencies factors. The obtained result using the proposed technique is fast, more accurate, and has not harm effect to iris structure. It is suitable for improving IoT security and authentication. In future, iris encoding and identification over IoT server will be proposed.

\section{References}

[1] M. H. A. Abdelsamea, M. Zorkany, and N. Abdelkader, "Real Time Operating Systems for the Internet of Things, Vision, Architecture and Research Directions," in Proceedings - 2016 World Symposium on Computer Applications and Research, WSCAR 2016, 2016.

[2] J. A. Stankovic, "Research directions for the internet of things," IEEE Internet Things J., vol. 1, no. 1, pp. 3-9, 2014.

[3] J. Gubbi, R. Buyya, S. Marusic, and M. Palaniswami, "Internet of Things (IoT): A vision, architectural elements, and future directions," Futur. Gener. Comput. Syst., vol. 29, no. 7, pp. 1645-1660, 2013.

[4] Z. He, T. Tan, Z. Sun, and X. Qiu, "Toward accurate and fast iris segmentation for iris biometrics," IEEE Trans. Pattern Anal. Mach. Intell., vol. 31, no. 9, pp. 1670-1684, 2009.

[5] Navita Kamboj and P. Gupta, "A Review on Segmentation techniques for Iris Recognition System," Int. J. Eng. Manag. Res., vol. 5, no. 2, pp. 14-16, 2015.

[6] R. Gad, N. El-Fishawy, M. Zorkany, and A. El-SAYED, "FPGA Implementation of Iris Recognition Based on Fast DCT Coding," 25th International Conference on Computer Theory and Applications (ICCTA 2015). Alexandria, Egypt, pp. 185-190, 2015.

[7] R. M. Gad, M. A. Mohamed, and N. A. El-Fishawy, "Iris Recognition Based on Log-Gabor and Discrete Cosine Transform Coding," J. Comput. Sci. Eng., vol. 5, no. 2, pp. 19-26, 2011.

[8] Y. Lin, Z. Qu, Y. Zhang, and H. Han, "A Fast and Accurate Pupil Localization Method Using Gray Gradient Differential and Curve Fitting," in Proceedings of the 4th International Conference on Computer Engineering and Networks, 2015, pp. 495-503.

[9] Chunxian Song, Guangzhu Xu, Chunlin Li, and J. Jing, "Removing Speckles 
Selectively from Iris Images to Improve Pupil Location Using 2D Gabor Filters," Int. J. Hybrid Inf. Technol., vol. 5, no. 2, pp. 57-66, 2012.

[10] J. Daugman, "How Iris Recognition Works," IEEE Trans. Circuits Syst. Video Technol., vol. 14, no. 1, pp. 21-30, 2004.

[11] Ann A. Jarjes, Kuanquan Wang, and G. J. Mohammed, "Gvf Snake-based Method for Accurate Pupil Contour Detection," Inf. Technol. J., vol. 9, no. 8, pp. 1653-1658, 2010.

[12] Kien Nguyen Thanh, Clinton B. Fookes, and S. Sridharan, "Fusing Shrinking and Expanding Active Contour Models for Robust Iris Segmentation," 10th International Conference on Information Science, Signal Processing and their Applications. Renaissance Hotel, Kuala Lumpur, pp. 1-4.

[13] Walid Aydi, Nouri Masmoudi, and L. Kamoun, "New Corneal Reflection Removal Method Used In Iris Recognition System," Int. Sch. Sci. Res. Innov., vol. 5, no. 5, pp. 898-902, 2011.

[14] Pengfei Cai and C. Wang, "An Eyelid Detection Algorithm for the Iris Recognition," Int. J. Secur. Its Appl., vol. 9, no. 5, pp. 105-112, 2015.

[15] M. Patil and K. Raghuveer, "SVD and DWT Based Iris Recognition Using Beagleboard-xM," Int. J. Comput. Sci. Mob. Comput., vol. 4, no. 4, pp. 532 $540,2015$.

[16] S. S. Omran and A. A. Al-Hillali, "Quarter of Iris Region Recognition Using the RED Algorithm," in Proceedings of the 2015 17th UKSIM-AMSS International Conference on Modelling and Simulation, 2015, pp. 66-72.

[17] S. Umer, B. C. Dhara, and B. Chanda, "Texture code matrix-based multiinstance iris recognition," Pattern Anal. Appl., vol. 19, no. 1, pp. 283-295, 2016.

[18] R. Gad, M. Zorkany, A. EL-Sayed, and N. EL-Fishawy, "An Efficient Approach for Simple Iris Localization and Normalization Technique," Menoufia Journal of Electronic Engineering Research (MJEER), vol. 25, no. 2, pp. 213-224, 2016.

[19] Zhou Wang, Alan Conrad Bovik, Hamid Rahim Sheikh, and Eero P. Simoncelli, "Image Quality Assessment: From Error Visibility to Structural Similarity," IEEE Trans. Image Process., vol. 13, no. 4, pp. 600-612, 2004.

[20] O. G. E. MARQUES, Image and Video Processing Using MATLAB, vol. 1. Canada.: John Wiley \& Sons, 2011.

[21] Zhou Wang and Alan Conrad Bovik, "A Universal Image Quality Index," IEEE Signal Process. Lett., vol. 9, no. 1, pp. 81-84, 2002.

[22] R. P. Wildes, "Iris Recognition: An Emerging Biometric Technology," Proc. IEEE, vol. 85, no. 9, pp. 1348-1363, 1997.

[23] Ramadan Gad, Nawal El-Fishawy, M. Zorkany, and A. EL-SAYED, "FPGA Implementation of Iris Recognition Based on Fast DCT Coding," 25th 
International Conference on Computer Theory and Applications (ICCTA 2015). Alexandria, Egypt, pp. 185-190.

[24] "Chinese Academy of Sciences Institute of Automation (CASIA) v.1." [Online]. Available: http://biometrics.idealtest.org.

[25] "Chinese Academy of Sciences Institute of Automation (CASIA) v.4Interval." [Online]. Available: http://biometrics.idealtest.org.

[26] H. Proença and L. A. Alexandre, "UBIRIS: A noisy iris image database," in International Conference on Image Analysis and Processing, 2005, pp. 970977.

[27] Y. Yin, L. Liu, and X. Sun, "SDUMLA-HMT: a multimodal biometric database," in Chinese Conference on Biometric Recognition, 2011, pp. 260268.

\section{ملخص البحث بالثغة العربية}

\section{استخلاص فزحية العين عبر انترنت الأشباء}

يعتير انترنت الأشياء من التكنولوجيا المستحدثة و المتطورة. وتطبيقاتها بحاجة لإي

نظا حماية مثل بصدة قزحية العين لتأمين مثاركة البيانات و التحقق من المستخدمين. ولما

كانت مرحلة استخلاص الفزحية من الهـ المراحل في النظام وأصعبها، بالإضافة الي

المشاكل التي بحاجة اليي حلول مثل: التغطية بلرموش والجفون وانعكاسات الضوء في

منطقة البؤبؤ، فهذا البحث يقدم خوارزم| لحل هذد المشاكل. بِالاضافة اليـي قصور فقدان

البيانات وعو امل التقاط القزحية المختفة في ضوء اختلاف انظمة الصور ــ وأعطت النتائج

دقة عائة وزمن تنفيذ جيد بناسب تطبيقت انترنت الأشياء. معلي نجاح بدقة اكبر من

99.545 ٪ وزمن تنفيذ في اسيوأ الحالات 0.758 ثانية. وبذلت بسهل تحقيق حماية

التحقى من البيانات والاشخاص عن استخدام هذا الخيوايزح عبر انترنت الاشيباء. 